\title{
Mirroring Doubly Excited Resonances in Argon
}

\author{
S. E. Canton-Rogan, ${ }^{1}$ A. A. Wills, ${ }^{1}{ }^{1 *}$ T. W. Gorczyca, ${ }^{1}$ M. Wiedenhoeft, ${ }^{1}$ \\ O. Nayandin, ${ }^{1}$ Chien-Nan Liu, ${ }^{2}$ and N. Berrah ${ }^{1, \dagger}$ \\ ${ }^{1}$ Department of Physics, Western Michigan University, Kalamazoo, Michigan 49008 \\ ${ }^{2}$ Max-Planck-Institut für Physik komplexer Systeme, Nöthnitzer Strasse 38, D-01187 Dresden, Germany
}

(Received 3 April 2000)

\begin{abstract}
New features are revealed in the low-energy photoionization spectrum of Ar by critically combining high photon resolution and differential photoelectron spectroscopic techniques. Two $L S$-forbidden doubly excited resonances are seen in the $3 p_{3 / 2,1 / 2}^{-1}$ partial cross sections which exhibit mirroring profiles, resulting in complete cancellation in the total photoionization cross section, as was predicted by Liu and Starace [Phys. Rev. A 59, R1731 (1999)]. These results demonstrate that a new class of weakly spinorbit induced, mirroring resonances should be observable in partial, but not in total, collisional cross sections involving atoms, molecules, and solids in general.
\end{abstract}

PACS numbers: $32.80 . \mathrm{Fb}$

The early measurements of the low-energy photoionization spectrum in $\mathrm{Ar}$,

$$
h \nu+3 p^{6} \rightarrow 3 p^{5}+e^{-},
$$

using first-generation synchrotron radiation sources, were able to delineate windowlike resonances due to the singly excited $3 s^{-1} n p\left({ }^{1} P_{1}\right)$ Rydberg series [1], and later were also able to identify two low-lying, doubly excited resonances, $3 s^{2} 3 p^{4} 4 s\left({ }^{2} P_{1 / 2,3 / 2}\right) 4 p\left({ }^{1} P_{1}\right)$ [2]. Despite more than three decades of subsequent investigations, however, there has been no evidence of any other photoionization resonances in the energy region below the $\mathrm{Ar}^{+} 3 s^{-1}$ threshold.

The recent availability of third-generation sources [3], such as the Advanced Light Source, has brought a new level of sophistication to many areas of synchrotron radiation based research. Improvements in flux and resolving power now allow more precise measurements of the differential, partial photoionization cross sections. These are expressible as [4]

$$
\frac{d \sigma_{i}}{d \Omega}=\frac{\sigma_{i}}{4 \pi}\left[1+\beta_{i} P_{2}(\cos \theta)\right]
$$

where $\theta$ is the angle between the electron momentum and the photon polarization vector, $\sigma_{i}$ are the angle-integrated partial cross sections, and $\beta_{i}$ are the asymmetry parameters. Determination of $\sigma_{i}$ and $\beta_{i}$ through differential, partial measurements therefore completely characterizes the photoionization spectrum.

In this Letter, we report a highly detailed study of the low-lying resonances in the $\operatorname{Ar} 3 p_{3 / 2,1 / 2}^{-1}$ continua in the spectral range $26.4 \leq h \nu \leq 29.4 \mathrm{eV}$. Two new doubly excited, predominantly triplet resonances are observed which belong to a class of mirroring resonances [5] giving equal and opposite resonant contributions to the individual partial cross sections. As a result, their net contribution to the total cross section vanishes, which is why they are not observable in photoabsorption experiments [2,6,7]. These resonances have not been detected in previous measure- ments of the partial cross sections [8,9] since the achievable resolution was insufficient for resolving the rather narrow features $(<3 \mathrm{meV}$ FHWM) that can now be seen. Although we focus on photoionization of atomic argon, this phenomenon applies to general collisional processes in atoms, molecules, solids, etc.

The experiment was performed at the Advanced Light Source on the high flux and high-resolution atomic and molecular undulator beam line 10.0.1. A photon resolution of $3 \mathrm{meV}$ was achieved, close to the theoretical 10000 resolving power available at this beam line. Two complementary experimental setups were used to measure partial differential cross sections. In the first one, a spectrometer with a hemispherical analyzer fitted with an ISL (Integrated Sensors Ltd.), position sensitive detector [10] was employed to record photoelectron spectra at the magic angle $\left(\theta=54.7^{\circ}\right)$, giving the partial cross sections and the corresponding branching ratio. In the second one, two time-of-flight analyzers, at $\theta=0^{\circ}$ and $\theta=90^{\circ}$ with respect to the light polarization axis, were used to determine asymmetry parameters [11].

In both experiments, the data were collected with a twodimensional acquisition technique [12,13], consisting of a systematic accumulation of photoelectron spectra at many, close, and equally spaced photon energies, from which constant ionic state (CIS) spectra were extracted. The spectra were corrected for variations in incident light intensity. The electron transmission efficiency curves were slowly varying functions of energy and were assumed constant over the spectral range corresponding to the separation of the photoelectron lines $(177 \mathrm{meV})$. The photon energy scale was calibrated using the positions of the $3 s^{-1} n p$ window resonances by comparison with photoabsorption work $[2,6]$.

The CIS spectra of the two final states $3 p_{3 / 2,1 / 2}^{-1}$ are presented in Fig. 1 for all three angles. At $\theta=54.7^{\circ}$, their sum is proportional to the total ionization cross section. In addition to the indicated $3 s^{-1} n p\left({ }^{1} P_{1}\right)$ Rydberg series, the two previously reported low-lying doubly excited 


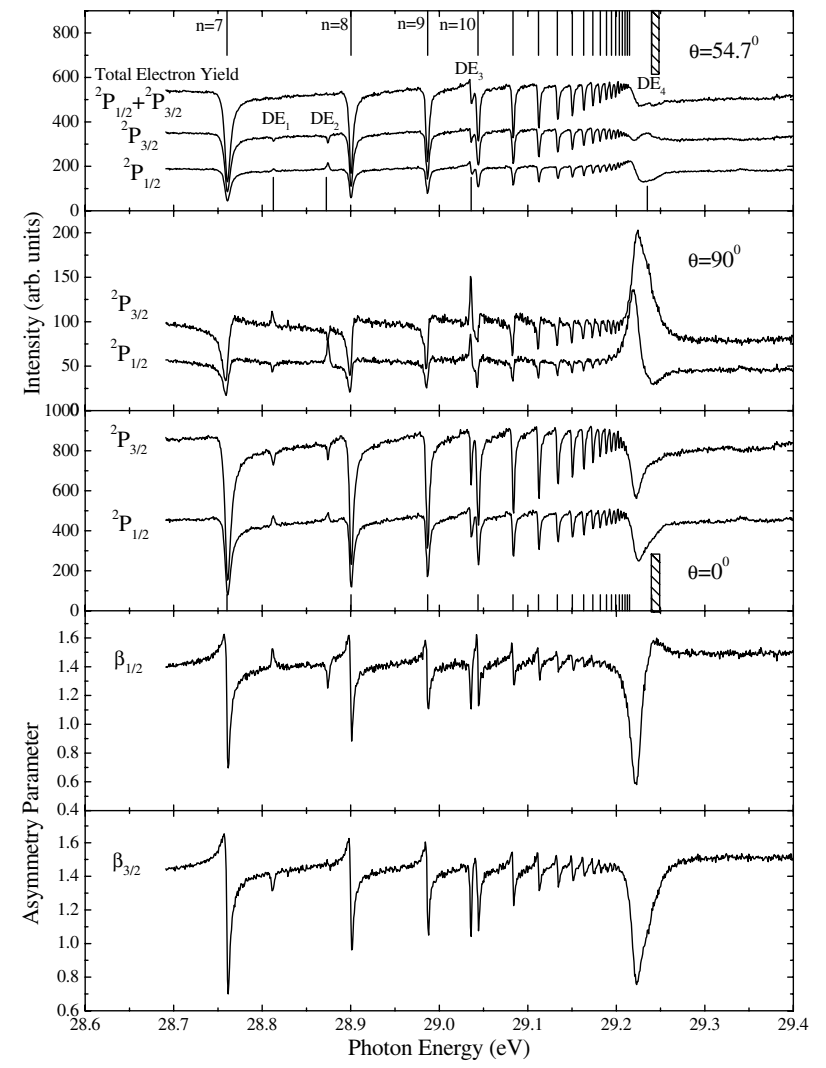

FIG. 1. Partial differential cross sections $\frac{d \sigma_{i}}{d \Omega}$ at $\theta=54.7^{\circ}$, $\theta=90^{\circ}$, and $\theta=0^{\circ}$, and asymmetry parameters $\beta_{i}$.

resonances $D E_{3}$ and $D E_{4}$, assigned, respectively, as $3 s^{2} 3 p^{4} 4 s\left({ }^{2} P_{3 / 2}\right) 4 p\left({ }^{1} P_{1}\right)$ and $3 s^{2} 3 p^{4} 4 s\left({ }^{2} P_{1 / 2}\right) 4 p\left({ }^{1} P_{1}\right)$ [2], are clearly seen. While there are no other observable resonances in the total cross section, the partial cross sections reveal two new resonances, $D E_{1}$ and $D E_{2}$, with mirroring profiles between the $3 s^{-1} 7 p$ and $3 s^{-1} 8 p$ members. They are further characterized by the measurements of the asymmetry parameters $\beta_{1 / 2}$ and $\beta_{3 / 2}$ (Fig. 1). Although similar mirroring behavior has been reported in partial photoionization cross sections of atoms [14-16], and very recently in photodetachment of negative ions [17], those observations were considered coincidental until the analytic developments of Liu and Starace [5] showed that this mirroring behavior is guaranteed under certain conditions, which we now consider.

Resonances in the total cross section can be understood based on the well-known parametrization of line profiles $[18,19]$. In the vicinity of an isolated resonance, the total photoabsorption cross section is characterized by an energy dependence $[18,19]$

$$
\sigma(\epsilon)=\sigma_{a} \frac{(q+\epsilon)^{2}}{1+\epsilon^{2}}+\sigma_{b},
$$

where $\sigma_{a}\left(\sigma_{b}\right)$ is the direct cross section from the atomic ground state to the continuum subspace that does (does not) interact with the resonance, and $\epsilon=\left(E-E_{r}\right) / \frac{1}{2} \Gamma$ indicates the departure of the incident photon energy $E$ from the resonance energy $E_{r}$, scaled by the resonance half-width $\frac{1}{2} \Gamma$. The so-called Fano $q$ parameter determines the overall profile, ranging from near-Lorentzian $(q \rightarrow \infty)$, to windowlike $(q=0)$, to completely asymmetric $(q= \pm 1)$.

Another parameter of present importance is the ratio $\rho^{2}=\sigma_{a} /\left(\sigma_{a}+\sigma_{b}\right)$, which characterizes the fractional depth of the minimum of the cross section $\left(\sigma_{a}\right)$ to the nonresonant value $\left(\sigma_{a}+\sigma_{b}\right)$. It can equivalently be considered as a measure of the oscillator strength to the continuum space interacting with the resonance compared to the total direct oscillator strength. Based on the generalized expressions for the partial cross sections in the vicinity of an isolated resonance [20], it was proved analytically that in the limit $\rho^{2} \rightarrow 0$, any two groupings [21] of partial cross sections mirror each other [5], i.e., an increase in one cross section is matched by a decrease in the other. Summing all partial cross sections, one gets the total cross section [5]:

$$
\sigma(\epsilon)=\left(\sigma_{a}+\sigma_{b}\right)\left(1+\frac{\rho^{2} q^{2}}{1+\epsilon^{2}}\right) .
$$

The asymmetric resonant energy dependence cancels in the total cross section, resulting in a symmetric Lorentzian profile. In the opposite limit $\rho^{2} \rightarrow 1$, while the total cross section exhibits single-channel-like behavior, any two groupings of partial cross sections mimic each other [22]. Although the analytic developments of Liu and Starace [5] were concerned with photoinduced atomic processes in particular, they were based on earlier work of Fano [18] and Starace [20] which applies to general transitions; hence, these results are equally applicable to other collisional processes involving molecules, ions, solids, etc.

The cross sections displayed in Fig. 1 provide some examples of resonant behavior associated with different $\rho^{2}$ values, conveniently including the two extremes $\rho^{2} \rightarrow 0$ and $\rho^{2} \rightarrow 1$, and an intermediate case. The entire $3 s^{-1} n p\left({ }^{1} P_{1}\right)$ series and the doubly excited state $\left(D E_{3}\right)$ exhibit deep minima, indicating a $\rho^{2} \simeq 1$ character, and indeed the observed individual partial cross sections mimic each other. The broad and weak resonance $D E_{4}$ near the $3 s^{-1}$ threshold is the result of the partially mirroring structure in each CIS spectrum, so it has an intermediate $\rho^{2}$ value.

In light of the dramatic difference between the profiles of $D E_{3}$ and $D E_{4}$, the original assignment as $3 s^{2} 3 p^{4} 4 s\left({ }^{2} P_{1 / 2,3 / 2}\right) 4 p\left({ }^{1} P_{1}\right)$ might at first seem surprising. However, the local spectral landscape where they are found is also quite different: whereas $D E_{3}$ is only close to the $3 s^{-1} 10 p$ resonance, $D E_{4}$ is superimposed on unresolved high-lying members of the $3 s^{-1} n p\left({ }^{1} P_{1}\right)$ series and the $3 s^{-1} \epsilon p$ continuum, giving a broader width.

The mirroring resonances show no observable fractional depth in the total cross section, which is characteristic of $\rho^{2} \simeq 0$ resonances. More specifically, these examples of exact mirroring correspond to the case of $\rho^{2} q^{2} \simeq 0$ [5], which shows no resonance peak at all. They further 
illustrate that partial cross sections are more sensitive to resonances than the corresponding total cross section, in which resonant contributions may cancel out.

To our knowledge, the two mirroring resonances have not been predicted or observed before. Considering the classification of the $D E_{3}$ and $D E_{4}$ resonances at 29.03 and $29.23 \mathrm{eV}$ as $4 p$ valence orbitals coupled to the $3 s^{2} 3 p^{4}\left({ }^{3} P\right) 4 s\left({ }^{2} P_{3 / 2,1 / 2}\right)$ ionic states [2], any lower resonances, such as the newly observed ones $D E_{1}$ and $D E_{2}$ at 28.81 and $28.87 \mathrm{eV}$, must, in fact, be described by a configuration with a valence $4 p$ orbital coupled to a lowerenergy ionic parent. Relative energies of the few lowest $\mathrm{Ar}^{+}$ionic states are listed in Table I, where it is clearly seen that the parent must be either the $3 s^{2} 3 p^{4}\left({ }^{3} P\right) 3 d\left({ }^{4} D\right)$ state or the $3 s^{2} 3 p^{4}\left({ }^{3} P\right) 4 s\left({ }^{4} P\right)$ state. Coupling a $4 p\left({ }^{2} P^{o}\right)$ electron to either parent gives a resultant resonance of minimum spin 1 (triplet), which is $L S$ forbidden in a transition from the singlet ground state of Ar. Consequently, likely candidates could be assigned the main $L S$ configurations $3 d\left({ }^{4} D\right) 4 p\left({ }^{3} P_{1}\right), 3 d\left({ }^{4} D\right) 4 p\left({ }^{3} D_{1}\right), 4 s\left({ }^{4} P\right) 4 p\left({ }^{3} P_{1}\right)$, and $4 s\left({ }^{4} P\right) 4 p\left({ }^{3} D_{1}\right)$, with a common $3 p^{4}\left({ }^{3} P\right)$ core. An exact assignment of these two resonances requires accurate theoretical results, which are not yet available [24].

We now consider photoexcitation to $L S$-forbidden states, and how this relativistic effect results in the observed mirroring. In the absence of relativistic effects, the ratio of partial cross sections $r=\sigma_{3 / 2} / \sigma_{1 / 2}$ is equal to the statistical value $r=2$, on or off resonance [25]. However, nonstatistical branching ratios for photoionization of rare gases have been known for a long time $[14,26]$. As seen in Fig. 2, our measured ratio is somewhat less than two throughout the spectral range covered, and undergoes rapid variations in the vicinity of the resonances. More specifically, the slight departure from 2 off resonance $(r \simeq$ 1.9 ) is the signature of a small (few percent) mixing between the $L S$-allowed $3 s^{2} 3 p^{5} \epsilon s, \epsilon d\left({ }^{1} P_{1}\right)$ continua and the $L S$-forbidden $3 s^{2} 3 p^{5} \epsilon s, \epsilon d\left({ }^{3} P_{1},{ }^{3} D_{1}\right)$ continua, emphasizing that $L S$ coupling is no longer suitable to describe the symmetry of the system. Since the triplet resonances

TABLE I. Energies of the lowest $\mathrm{Ar}^{+}$states.

\begin{tabular}{lccc}
\hline \hline Configuration & Term & $J_{c}$ & Energy (eV) [23] \\
\hline $3 s^{2} 3 p^{5}$ & ${ }^{2} P^{o}$ & $3 / 2$ & 0.00000 \\
& & $1 / 2$ & 0.17749 \\
$3 s 3 p^{6}$ & ${ }^{2} S$ & $1 / 2$ & 13.47975 \\
$3 s^{2} 3 p^{4}\left({ }^{3} P\right) 3 d$ & ${ }^{4} D$ & $7 / 2$ & 16.40650 \\
& & $5 / 2$ & 16.42558 \\
& & $3 / 2$ & 16.44412 \\
& & $1 / 2$ & 16.45738 \\
$3 s^{2} 3 p^{4}\left({ }^{3} P\right) 4 s$ & ${ }^{4} P$ & $5 / 2$ & 16.64386 \\
& & $3 / 2$ & 16.74853 \\
& & $1 / 2$ & 16.81247 \\
$3 s^{2} 3 p^{4}\left({ }^{3} P\right) 4 s$ & ${ }^{2} P$ & $3 / 2$ & 17.14003 \\
& & $1 / 2$ & 17.26583 \\
\hline \hline
\end{tabular}

$D E_{1}$ and $D E_{2}$ autoionize primarily to (predominantly) triplet continua, which have very weak oscillator strengths from the singlet ground state, $\sigma_{a}=\sigma_{\text {triplet }} \ll \sigma_{\text {singlet }}=$ $\sigma_{b} \quad\left(\rho^{2} \simeq 0\right)$, and mirroring behavior is guaranteed. Hence, these resonances are observable in the partial cross sections but not in the total cross section. The present high-resolution experiment was therefore essential for observing these subtle effects.

While the present example involves $L S$-forbidden transitions, mirroring behavior among partial cross sections in the vicinity of a resonance are common occurrences in the high-energy part of photofragmentation spectra, where the fractional depths of the resonances are usually small. However, mirroring behavior could be suppressed if the resonance profiles in the partial cross section are dominated by their symmetric component $\frac{\rho^{2} q^{2}}{1+\epsilon^{2}}$. Nevertheless, resonances in different partial cross sections are expected to show different asymmetries. For example, in a recent experimental measurement of Li photoionization, the resonance profiles of the $2 s^{2} 2 p$ triply excited state in the single and double ionization partial cross sections exhibit $q$ values of different signs [27].

The important implication of mirroring behavior is that the intrinsic interference effects due to two indistinguishable quantum paths are not negligible, even though the symmetric resonance profiles suggest otherwise. For example, in studies of resonant Auger processes, a two-step sequential model has been commonly used in describing

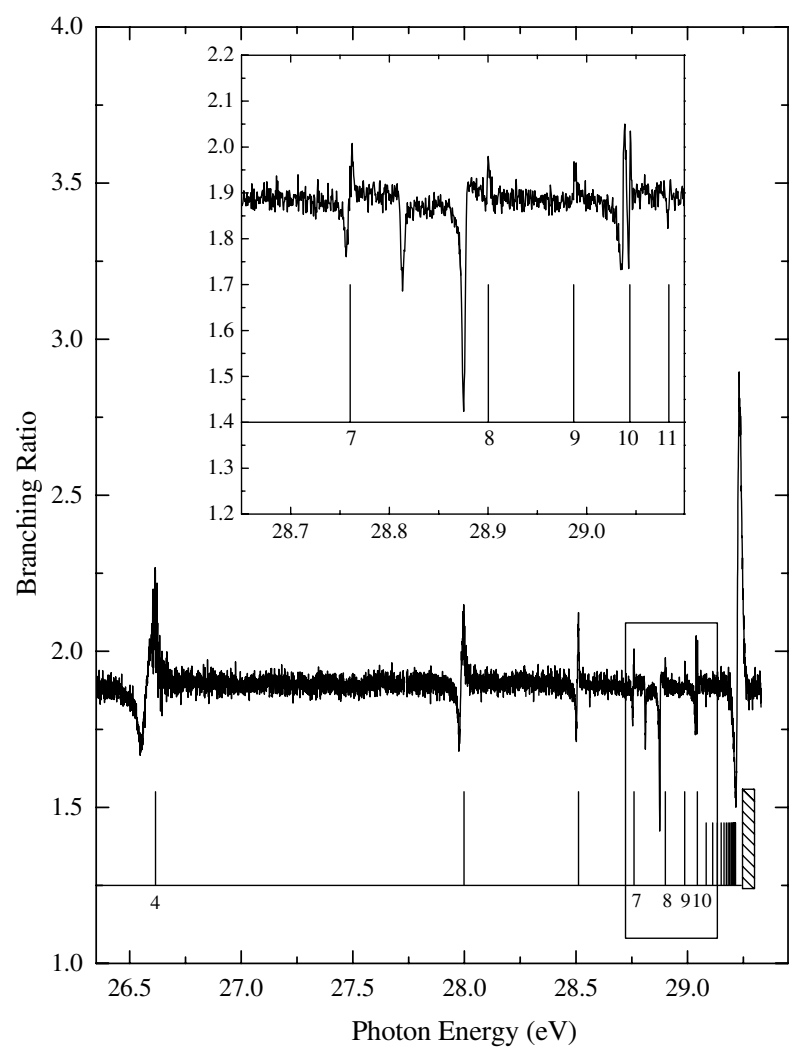

FIG. 2. Ratio of partial cross sections $r=\frac{\sigma_{3 / 2}}{\sigma_{1 / 2}}$. 
the symmetric resonance profiles observed in the total cross section. However, recent theoretical and experimental measurements show asymmetric resonance profiles in the partial cross sections as well as mirroring behavior among different partial cross sections [28]. Therefore, using a two-step sequential model to describe the autoionization resonances in photofragmentation processes does not give a correct picture, even if the resonance profile in the total cross section is Lorentzian. The present results illustrate that intrinsic interference effects involved in resonant photofragmentation processes are not negligible, even if the symmetric resonance profiles in the total cross section suggest a small interference effect.

In conclusion, we have observed two new mirroring resonances in the low-energy photoionization spectrum of Ar that are undetectable in the total photoionization cross section. They belong to a class of resonances predicted by Liu and Starace for the limiting case $\rho^{2} \rightarrow 0$. Analysis of differential parameters shows that the observed mirroring behavior is related to the $L S$-forbidden nature of these predominantly triplet resonances. Of broader implication, similar hidden resonances should be observable in other systems, and are not just limited to photoinduced transitions in atoms, but include collisional transitions in ions, molecules, and solids, for instance. The present results show that highly differential studies are necessary to observe certain important spectral features, and provide useful guidelines and stringent tests for the inclusion of electron correlation and relativistic effects in atomic calculations.

Discussions with C.H. Greene, H. van der Hart, and A.F. Starace are appreciated. This work was supported by the U.S. Department of Energy, Office of Science, Basic Energy Science, Division of Chemical Science, under Contract No. DE-FG02-92ER14299.

*Present address: Fritz-Haber-Institut der MPG, Faradayweg 4-6, D14195 Berlin, Germany.

†Electronic address: berrah@wmich.edu

[1] R. P. Madden and K. Codling, Phys. Rev. Lett. 10, 516 (1963).

[2] R. P. Madden, D. L. Ederer, and K. Codling, Phys. Rev. 177, 136 (1969).

[3] V. Schmidt, Rep. Prog. Phys. 55, 1483 (1992).

[4] A.F. Starace, Theory of Atomic Photoionization, Handbuch der Physik, edited by W. Mehlhorn (Springer, Berlin, 1982), pp. 1-121.

[5] C. N. Liu and A. F. Starace, Phys. Rev. A 59, R1731 (1999).
[6] M. A. Baig and M. Ohno, Z. Phys. 3, 369 (1986).

[7] N. Berrah, B. Langer, J.D. Bozek, T. W. Gorczyca, O. Hemmers, D. W. Lindle, and O. Toader, J. Phys. B 29, 5351 (1996).

[8] K. Codling, J.B. West, A.C. Parr, J.L. Dehmer, and R. Stockbauer, J. Phys. B 13, L693 (1980).

[9] A. Svensson, M. O. Krauss, and T. A. Carlson, J. Phys. B 20, L271 (1987).

[10] J. V. Hatfield, S. A. Burke, J. Comer, F. Currell, J. Goldfinch, T. A. York, and P. J. Hicks, Rev. Sci. Instrum. 63, 235 (1992).

[11] M. Wiedenhoeft, A. A. Wills, S. Canton, O. Nayandin, J. Viefhaus, and N. Berrah, in Abstracts of the 18th International Conference on X-ray and Inner-Shell Processes, Chicago, 1999, p. 177.

[12] E. Sokell, A. A. Wills, D. Cubric, F. J. Currell, J. Comer, and P. Hammond, J. Electron Spectrosc. Relat. Phenom. 94, 107 (1998).

[13] N. Berrah, B. Langer, A. A. Wills, E. Kukk, J. D. Bozek, A. Farhat, and T. W. Gorczyca, J. Electron Spectrosc. Relat. Phenom. 101, 1 (1999).

[14] J.A. R. Samson and R. B. Cairns, Phys. Rev. 173, 80 (1968).

[15] M. O. Krause, F. Cerrina, A. Fahlman, and T. A. Carlson, Phys. Rev. Lett. 51, 2093 (1983).

[16] M. G. Flemming, J.Z. Wu, C.D. Caldwell, and M.O. Krause, Phys. Rev. A 44, 1733 (1991).

[17] G. Haeffler, I. Yu. Kiyan, D. Hanstorp, and D. J. Pegg, Phys. Rev. A 57, 2216 (1998).

[18] U. Fano, Phys. Rev. 124, 1866 (1961).

[19] U. Fano and J. W. Cooper, Phys. Rev. 137, A1364 (1965).

[20] A. F. Starace, Phys. Rev. A 16, 231 (1977).

[21] By groupings, we mean that, given $N$ partial cross sections making up the total cross section, one group consists of the sum of any $M$ partial cross sections, and the complementary group consists of the sum of the other $N-M$ partial cross sections.

[22] C. N. Liu and A. F. Starace, Phys. Rev. A 60, 4647 (1999).

[23] Atomic Energy Levels, edited by C. E. Moore, Natl. Bur. Stand. U.S. Circular No. 467 (U.S. GPO, Washington, DC, 1949), Vol. I.

[24] Preliminary investigations of these photoexcited resonances using $R$-matrix and multiconfiguration Hartree-Fock techniques have encountered extreme difficulty in obtaining a converged description of their highly correlated (doubly excited) and spin-forbidden nature.

[25] See, for example, A. R. P. Rau, in Electron and Photon Interactions with Atoms, edited by $\mathrm{H}$. Kleinpoppen and M. R. C. McDowell (Plenum, New York, 1976), p. 141.

[26] J. A. R. Samson and A.F. Starace, J. Phys. B 8, 1806 (1975).

[27] R. Wehlitz, M. T. Huang, K. A. Berrington, S. Nakazaki, and A. Azuma, Phys. Rev. A 60, R17 (1999).

[28] T. W. Gorczyca and F. Robicheaux, Phys. Rev. A 60, 1216 (1999). 